\title{
ARTICLE Endogenous miRNA Sponge LincRNA-ROR promotes proliferation, invasion and stem cell-like phenotype of pancreatic cancer cells
}

\author{
Zhiqiang $\mathrm{Fu}^{1,2,7}$, Guolin $\mathrm{Li}^{1,2,7}$, Zhihua $\mathrm{Li}^{3,7}$, Yingxue Wang ${ }^{4}$, Yue Zhao ${ }^{5}$, Shangyou Zheng ${ }^{1,2}$, Huilin Ye ${ }^{1,2}$, Yuming Luo ${ }^{1,2}$, Xiaohui Zhao $^{2,3}$, \\ Lusheng Wei ${ }^{1,2}$, Yimin Liu ${ }^{6}$, Qing Lin ${ }^{1,2}$, Quanbo Zhou ${ }^{1,2}$ and Rufu Chen ${ }^{1,2}$
}

The long intergenic non-coding RNA, regulator of reprogramming (linc-ROR) is an oncogene and plays a key role in the embryonic stem cell maintenance and is involved in cancer progression. The objective of this study was to analyze linc-ROR expression in pancreatic ductal adenocarcinoma (PDAC) and determine the regulation effects of linc-ROR on proliferation and invasion of cancer cells, as well as properties of cancer stem-like cells (CSLCs). In this study, we found that linc-ROR was up-regulated in PDAC tissues and related to poor prognosis. Linc-ROR knockdown in pancreatic cancer cells inhibited cell growth and arrested in G1 phrase. Suppressed linc-ROR expression also attenuated cancer cell migration, invasion, and epithelial-mesenchymal transition. We observed that linc-ROR expression was increased in CSLCs. Importantly, linc-ROR knockdown impaired the properties and tumorigenesis of pancreatic CSLCs in vivo. Mechanistically, we found that linc-ROR functioned as a competing endogenous RNA (ceRNA) to several tumor suppressor microRNAs, particularly some members of let-7 family. We conclude that, as a crucial oncogene, linc-ROR promotes cell proliferation, invasiveness and contributes to stem cell properties of CSLCs in PDAC via acting as a ceRNA to regulate function of microRNAs. The linc-ROR is a potential therapeutic target for PDAC.

Cell Death Discovery (2017) 3, 17004; doi:10.1038/cddiscovery.2017.4; published online 29 May 2017

\section{INTRODUCTION}

Pancreatic ductal adenocarcinoma (PDAC) holds one of the most malignant types of human cancer. Despite tremendous efforts, PDAC is still related to a short survival with about 7 percent fiveyear survival rate now. Obviously, it is important to further understand the mechanism of PDAC development and dig innovative therapy approaches. In recent years, it is well acknowledged that non-coding RNAs (ncRNAs) play a vital role in both normal physiology and diseases. ${ }^{1}$ Moreover, long noncoding RNAs (IncRNAs), including antisense IncRNA, Intronic transcript, large intergenic noncoding RNA (lincRNA), promoterassociated IncRNA and UTR associated IncRNA, defined as ncRNA $>200$ nucleotides in length, are attracting tremendous attention as its important role in regulating vital cellular functions. ${ }^{1}$ So far, a large range of function of IncRNAs has been identified, such as regulation of apoptosis and invasion, ${ }^{2}$ remolding of induced pluripotent stem cells (iPS), ${ }^{3}$ management of tissue differentiation, ${ }^{4}$ and grasp of cell fate. ${ }^{5}$ Importantly, many IncRNAs have been identified as being cancer-specific, ${ }^{6,7}$ these IncRNAs might be employed as novel biomarkers or therapeutic targets.

Besides microRNAs, a significant portion of the non-coding RNAs, including long non-coding RNAs and pseudogenes, harbors miRNA-response elements (MRE). ${ }^{8}$ Recent studies described a novel interplay among non-coding RNAs harboring MRE. These
MRE-harboring non-coding RNA transcripts act as competing endogenous RNAs (ceRNAs) that compete for a common pool of miRNAs and regulate the expression of protein-coding RNAs. ${ }^{9}$ In previous reports, a muscle-specific LncRNA MD1 has been reported to control muscle differentiation by functioning as a ceRNA targeting miR-133. ${ }^{4}$ In addition, the non-coding $3^{\prime} U T R$ of HMGA2 transcript can disturb let-7 activity by changing miRNA targeting genes in lung cancer. ${ }^{10}$ In addition, InCRNA HULC, as a ceRNA, down-regulates a series of miRNAs, including miR-372 and thus promotes liver cancer development. ${ }^{11}$ The ceRNA mechanism links IncRNAs and miRNAs in the post-transcriptional network of tumor pathogenesis, which may explain disease processes and present opportunities for new therapies. However, its role in pancreatic cancer remains unknown.

Linc-ROR was first discovered as a sponsor of remolding of human $\mathrm{iPS}$, where it was previously hypothesized to promote the transcription of core pluripotency factors including Oct4, Sox2 and Nanog based on the chromosome-modifying functions of many other reported lincRNAs. ${ }^{3,12}$ But recent studies showed that lincROR actually functions as a microRNA sponge for miR-145 to increase the expression of mir-145 targets, such as OCT4, SOX2 and Nanog. ${ }^{12,13}$ Moreover, linc-ROR has been demonstrated to make an important impact in the regulation of hypoxia signaling pathways in liver original cancer cells. ${ }^{14}$ In breast cancer, Hou et al.

\footnotetext{
${ }^{1}$ Department of Hepato-Pancreato-Billiary Surgery, Sun Yat-sen Memorial Hospital, Sun Yat-sen University, Guangzhou, China; ${ }^{2}$ Guangdong Provincial Key Laboratory of Malignant Tumor Epigenetics and Gene Regulation, Medical Research Center, Sun Yat-sen Memorial Hospital, Sun Yat-sen University, Guangzhou, China; ${ }^{3}$ Department of Medical Oncology, Sun Yat-sen Memorial Hospital, Sun Yat-sen University, Guangzhou, China; ${ }^{4}$ Department of Endocrinology, The First Affiliated Hospital, Jinan University, Guangzhou, China; ${ }^{5}$ Department of Gastroenterology, The First Affiliated Hospital, Sun Yat-sen University, Guangzhou, China and ${ }^{6}$ Department of Radiotherapy, Sun Yat-sen Memorial Hospital, Sun Yat-sen University, Guangzhou, China.

Correspondence: Q Zhou (zhouquanbo8133@163.com) or R Chen (chenrufu8133@163.com)

${ }^{7}$ These authors contributed equally to this work.

Received 29 November 2016; revised 24 December 2016; accepted 29 December 2016; Edited by A Rufini
} 
reported that lincRNA-ROR could induce epithelial-tomesenchymal transition (EMT) and promote carcinogenesis and development of breast cancer by targeting miR-205. ${ }^{15}$ As many embryonic stem cells-related genes often play a similar function in cancer stem cells (CSCs), ${ }^{16,17}$ it is valid to hypothesize that lincROR may also implement a role in regulating CSCs properties. In addition, the fact that linc-ROR can act as a sponge not only for miR-145 but also for let- $7,{ }^{15}$ miR-205 ${ }^{15}$ and other potential miRNAs, ${ }^{12}$ and findings that other IncRNAs with ceRNA activity usually have several target miRNAs, ${ }^{9}$ attracted us to speculate that linc-ROR may also affect CSCs properties through regulating one or more important tumor-suppressor miRs.

In this study, we aimed to explore the role of linc-ROR in regulation of proliferation, invasion and the CSC properties of cancer stem-like cells (CSLCs) in pancreatic cancer. We showed that linc-ROR knock-down impaired the proliferation, colony formation, migration, invasion ability and decreased the expression of EMT-related genes. Moreover, we identified an important role of linc-ROR in the maintaining of CSC properties of CSLCS in pancreatic cancer cells. Furthermore, we found that let-7, miR-7 and miR-451, which have been known to have key roles in repressing tumor proliferation, invasion and CSLCs properties, were ceRNA targets of linc-ROR. We thus identify an important and novel regulatory mechanism of linc-ROR in pancreatic cancer progression.

\section{RESULTS}

Linc-ROR is over-expressed in human PDAC tissues and related to poor prognosis

To investigate the role of linc-ROR in pancreatic cancer, we first evaluated the linc-ROR expression in paired tumor and para-tumor tissues from 81 clinical PDAC specimens. Quantitative realtimePCR(qRT-PCR) analysis demonstrated that linc-ROR expression was up-regulated in tumor tissues compared with para-tumor tissues (Figure 1a). In addition, log-rank analysis indicated that overall survival was significantly reduced in patients with higher linc-ROR expression $(P=0.047)$ (Figure $1 \mathrm{~b})$. These results indicated that increased level of linc-ROR may be positively correlated with the progression of pancreatic cancer.

\section{Linc-ROR regulates pancreatic cancer cell proliferation}

Stable linc-ROR knock-down cell lines (PANC-1 and SW1990) were established by using retrovirus infection, and the silencing effect was confirmed by qRT-PCR. To assess the role of linc-ROR in pancreatic cancer cell proliferation, we performed MTT assays on control and linc-ROR-suppressed cells. The depletion of linc-ROR expression reduced cell proliferation significantly compared with shControl cells both in PANC-1 cell line (Figure 2a) and SW1990 cell line (Figure 2b). We further analyzed the effect of linc-ROR on cell cycle, and found linc-ROR suppression obviously reduces number of cells in the S-phase and increase in the G0/G1 phase (Figures 2c and d). As expected, Knockdown of linc-ROR significantly decreased the clone formation of both cells (Figures 2e and f).

Linc-ROR regulates pancreatic cancer cell migration, invasion and EMT

We evaluated the effects of linc-ROR on cell migration and invasion of PANC-1 with relatively moderate level of linc-ROR expression, and on SW1990 cells which expresses relatively higher levels of linc-ROR. We first examined the effect of linc-ROR knockdown on PANC-1 and SW1990 cells migration using wound healing assay in the presence of Mitomycin, and found that cells stable transfected with linc-ROR shRNA had significant slower motility (relative wound closure proportion) compared with cells stable transfected with scramble RNA (Figures $3 a$ and b). Furthermore, we investigated whether suppression of linc-ROR expression would impair cell invasion ability. Matrigel invasion assay showed that stable knockdown of linc-ROR dramatically reduced the invasion of PANC-1 and SW1990 cells (Figure 3c). We next examined whether the silencing of linc-ROR expression can suppress the EMT process. Cells were seeded $1 \times 10^{6}$ per well on $6-$ well plate, and were incubated with medium containing $10 \mathrm{ng} / \mathrm{ml}$ transforming growth factor-beta (TGF- $\beta$ ) and $50 \mathrm{ng} / \mathrm{ml}$ stromalderived factor (SDF-1) for $72 \mathrm{~h}$. Western blot analyses showed that stable knock-down of linc-ROR was associated with impaired down-regulation of epithelial marker E-cadherin and up-regulation of mesenchymal markers $\mathrm{N}$-cadherin and Vimentin (Figure $3 \mathrm{~d}$ ). Furthermore, we examined the effect of up-regulating linc-ROR expression on other EMT-related genes by qRT-PCR, and results in contrast with the above findings are revealed (Figures $3 e$ and $f$ ).

\section{Linc-ROR is over-expressed in pancreatic CSLCs}

We obtained CSLCs from PANC-1 cells, as described in Materials and methods. The representative images of $\times 100$ light microscope view of spheres are shown in Figure 4a. As the sphereforming process is intended to enrich the potential CSLC subpopulations, we further detected the CSCs markers in spheres. Spheres were analyzed for the expression of previously reported CSLC-related markers and genes including CD44, CD133, ALDH1, SOX2 and Nanog. We found that the above markers are highly enriched in cells of spheres (Figures $4 \mathrm{~d}$ and e). In addition, the proportion of CD133 and ALDH1 double positive cells were dramatically increased in cells of spheres (Figures $4 \mathrm{~b}$ and $\mathrm{c}$ ). These data indicate that the CSLCs were enriched from PANC-1 cells through sphere formation. As linc-ROR was reported highexpressed in embryonic stem cells and pluripotent stem cells, we further examined the change of linc-ROR expression levels following sphere formation. As expected, the expression of
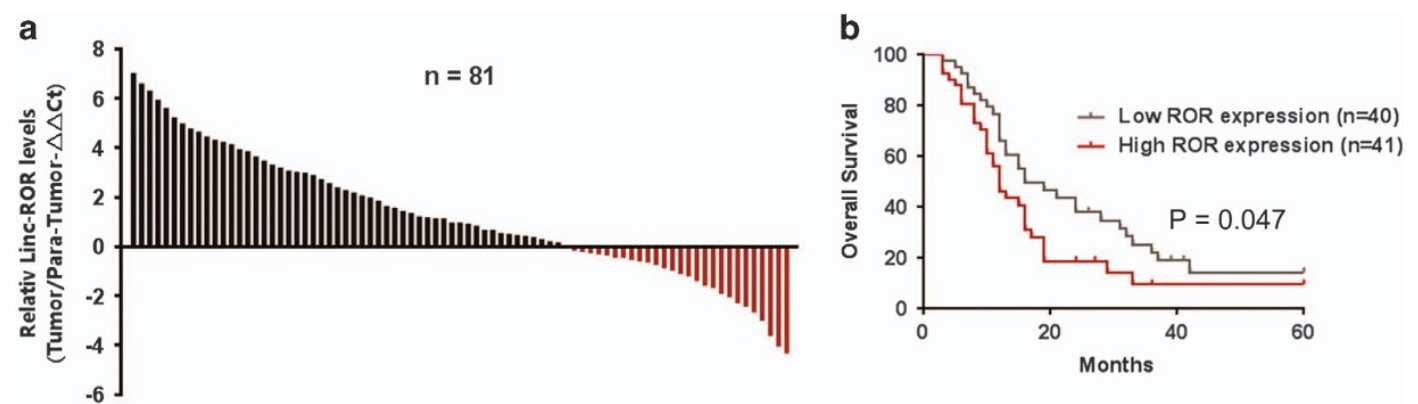

Figure 1. Linc-ROR is over-expressed in human PDAC tissues and is related to poor prognosis. (a) Relative expression levels of linc-ROR in 81 PDAC tissues compared with adjacent para-tumor tissues were evaluated by qRT-PCR. Results are shown as ${ }^{-\Delta \Delta}$ CT values. (b) The patients were divided into two groups based on linc-ROR levels. The log-rank test (two-sided) was used to compare differences between groups. The Kaplan-Meier curves show analyses of overall survival. 


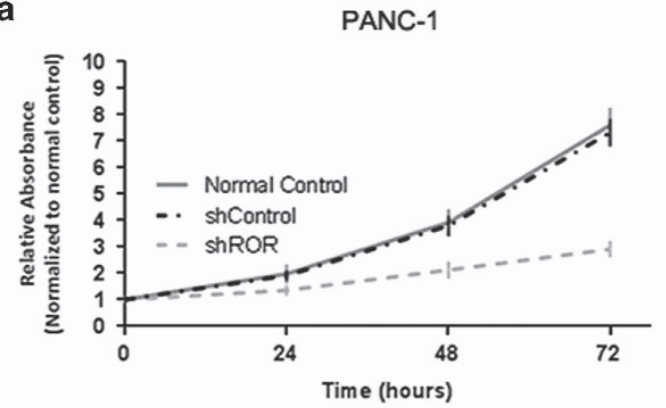

C

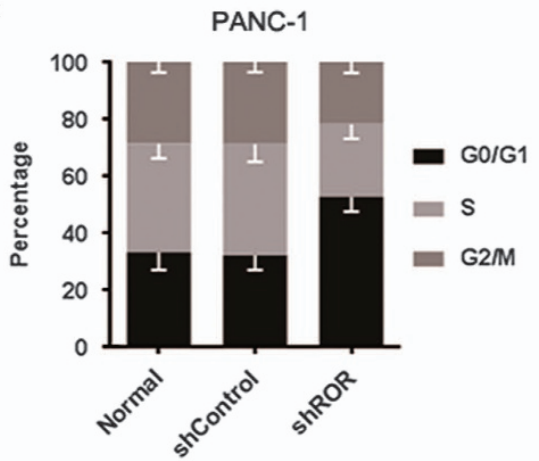

e
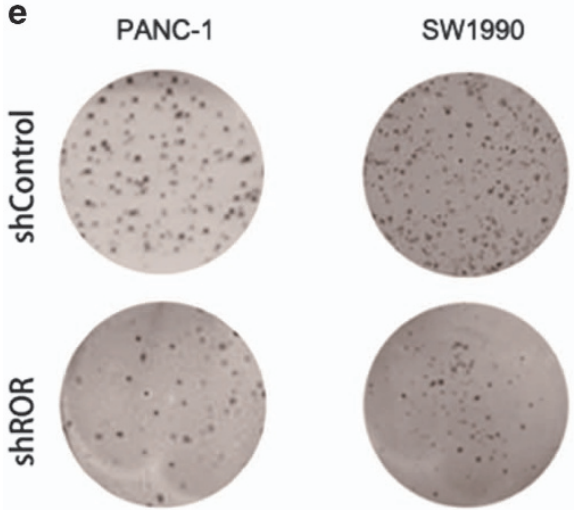

b

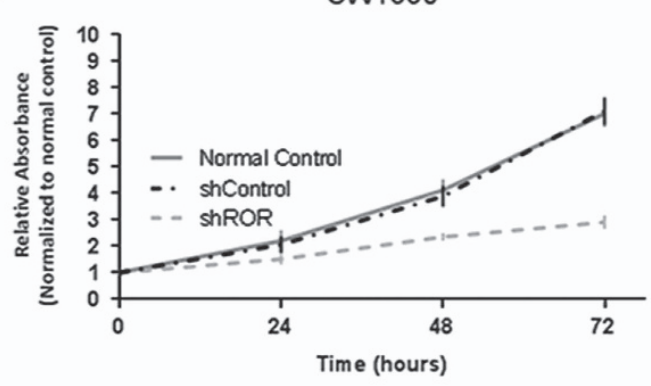

d

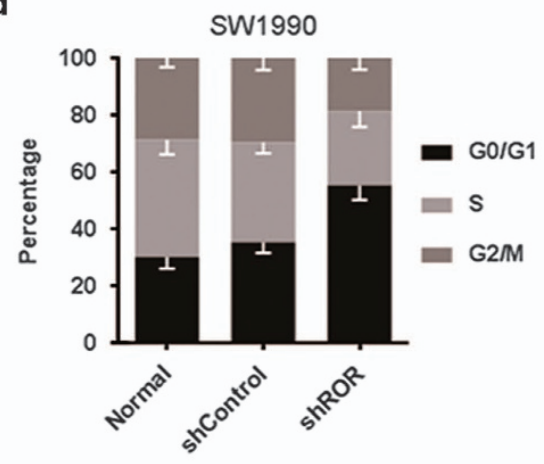

$\mathbf{f}$

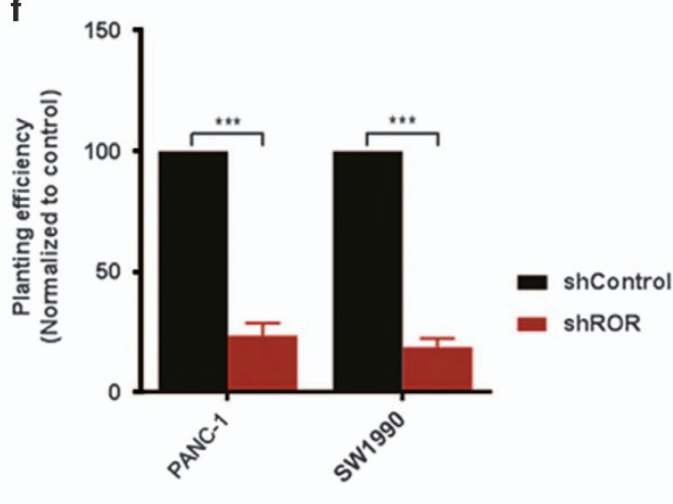

Figure 2. Linc-ROR regulates pancreatic cancer cell proliferation. (a) Results of MTT assays showing effect of linc-ROR knockdown on cell proliferation in PANC-1 cells. (b) Results of MTT assays showing effect of linc-ROR knockdown on cell proliferation in SW1990 cells. (c) Cell cycle analysis of PANC-1 cells and (d) SW1990 cells stably transfected with shRNA-mediated scramble (shControl) or shRNA-mediated silencing of linc-ROR (shROR). (e) Representative images of clone formation assays performed in PANC-1 cells and SW1990 cells transfected with shControl or shROR. (f) Colony formation was analyzed, the number of colonies of shROR group were normalized to that of shControl group. ErROR bars represent the mean \pm S.D. of triplicate experiments. Statistical significance was calculated using the Student's $t$ test or ANOVA tests. $* * * P<0.001$.

linc-ROR was increased 2.482 fold in spheres compared with control normal PANC-1 cells.

Linc-ROR regulates the CSC properties of stem cell-like pancreatic cancer cells

Given the important role of linc-ROR in maintaining stem cell properties, we next evaluated the effect of linc-ROR in regulating the stem cell-like properties of CSLCs derived in the above part. First, we compared the sphere formation ability between PANC-1 cells stably transfected with scramble RNA (shControl) and lincROR knock-down cells (shROR). We found that linc-ROR knockdown dramatically impaired the sphere formation of PANC-1 cells. The typical morphological feature of spheres derived from lincROR knock-down cells is shown in Figure 5a. The comparisons of total number of spheres derived from a same number of initial PANC-1 cells are shown in Figure 5b, a 10-fold reduction in formation number were observed in shROR cells. Also, the diameter of spheres derived from shROR cells was significantly decreased (Figure 5c). In addition, we picked spheres from both group, and examined the CSC markers in these cells by western blot and qRT-PCR, which demonstrated a decreased CSC markers in spheres derived from shROR cells (Figures $5 \mathrm{~d}$ and e). Furthermore, we selected spheres of $100-200 \mu \mathrm{m}$ in diameter from both group, and performed the secondary passage sphere formation test. After incubation in the sphere-culture medium about 10 days, the secondary passage spheres formed well by P1 cells of control group. In contrast, P1 cells with suppressed lincROR expression demonstrated impaired P2 sphere formation ability both in morphological features (Figure 5f), number (Figure $5 \mathrm{~g}$ ) and size (Figure $5 \mathrm{~h}$ ). At last, we evaluate the effect of linc-ROR on pancreatic CSLCs tumorigenicity. We analyzed the in vivo tumorigenicity of PANC-1 cells, cells of P1 spheres in nude mice (Figures $5 i$ and $j$ ). As expected, injection of $1 \times 10^{4}$ cells of P1 
a

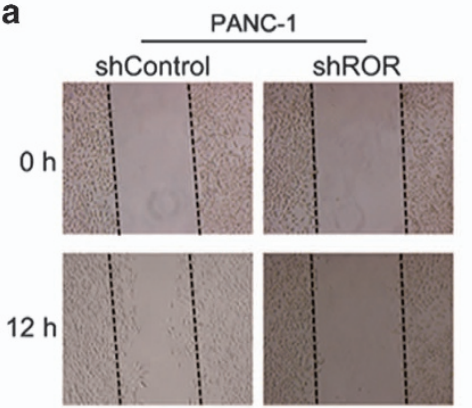

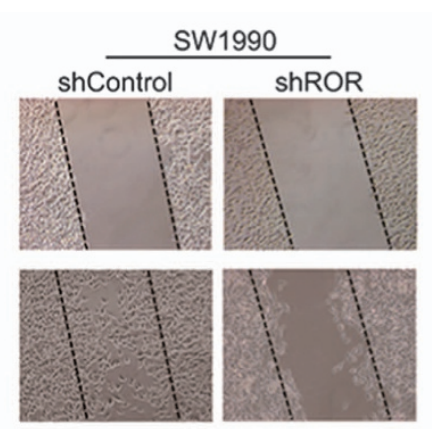

b

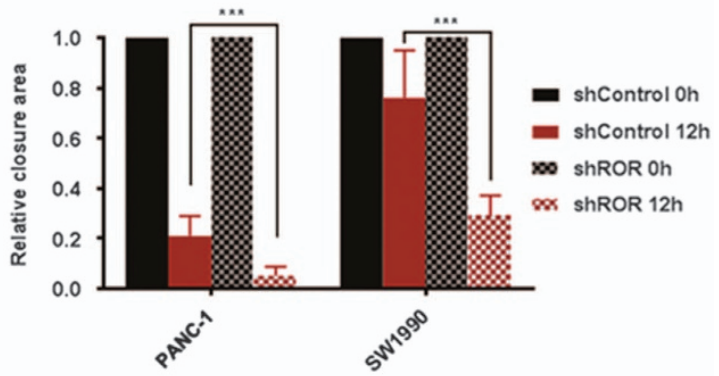

d

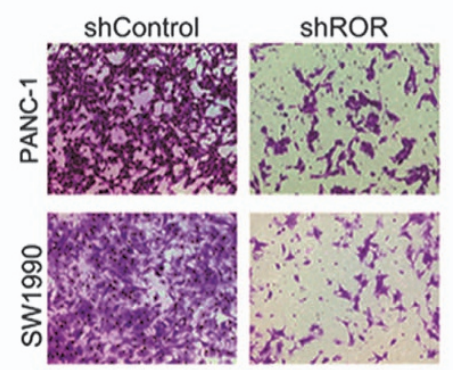

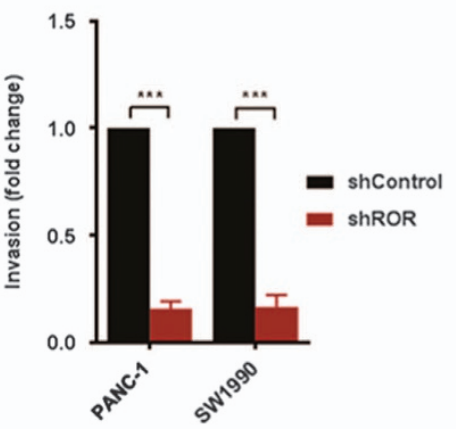

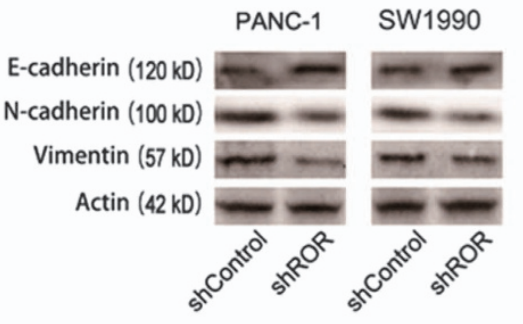

e

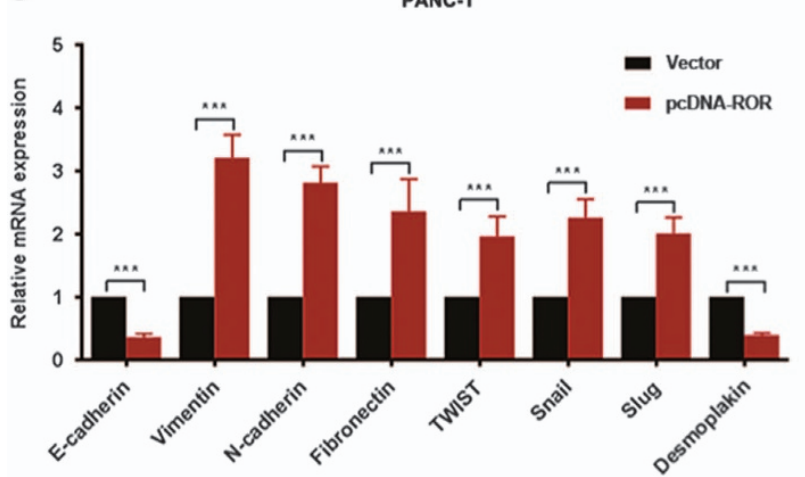

f

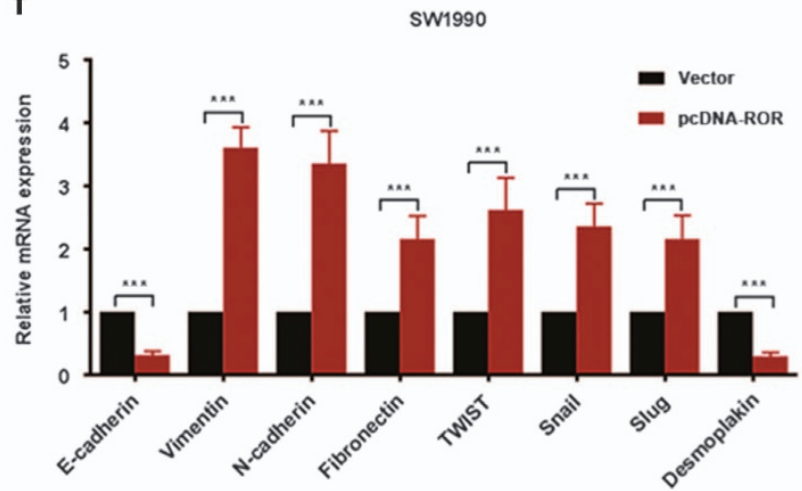

Figure 3. Linc-ROR regulates pancreatic cancer cell migration, invasion and EMT. (a) The scratch wound healing assay was performed in PANC-1 cells and SW1990 cells to assess the effect of linc-ROR on cell mobility in the presence of Mitomycin C. Dashed lines marked boundaries of the initial scratch. (b) Bars represented the percentage of wound healing, the area between dashed lines of shControls were set as 1. (c) Invasion assay were performed to assess the effect of linc-ROR on cell invasive ability of PANC-1 cells and SW1990 cells. Photos were representative fields of invasive cells on the membrane. (d) Western blot analysis of E-cadherin, N-cadherin and Vimentin was performed in PANC-1 cells and SW1990 cells stably transfected with shRNA-mediated scramble (shControl) or shRNA-mediated silencing of linc-ROR (shROR). (e) PANC-1 cells and (f) SW1990 cells were stably transfected with pCDNA3 empty vectors (NC) or pCDNA3 ROR overexpressing vectors (ROR), and the relative expression levels of mRNA expression of EMT-related genes were determined using qRT-PCR. Results are shown as ${ }^{2 \Delta \Delta}$ CT. ErROR bars represent the mean \pm S.D. of triplicate experiments. Statistical significance was calculated using the Student's $t$ test or ANOVA tests. ${ }^{* * *} P<0.001$.

spheres derived from PANC-1 cells stable transfected with scramble shControl RNA showed comparable tumorigenicity compared injection of $1 \times 10^{6}$ PANC- 1 cells stable transfected with scramble shControl RNA. In addition, as expected, P1 spheres derived from shROR PANC-1 cells demonstrated a much weaker tumorigenicity.

Linc-ROR functions as an endogenous microRNA Sponge in pancreatic cancer cells

It has been reported that linc-ROR can function as molecular sponges to bind relative miRNAs to affect their function, and down-regulating the expression of targeted-miRs. ${ }^{12}$ Therefore, the down-regulated miRs following sphere formation might be the potential target of linc-ROR. We employed microarray gene expression analysis (miRCURY LNA Array, v.18.0, Exiqon) using PANC-1 cells and spheres derived from PANC-1 cells (Supplementary Table 1). As a result, 123 miRs were found down-regulated more than two times in the cells of spheres. By comparing miRs have been reported to have inhibitory effect on stem properties of cancer cells (Supplementary Table 2), we found fourteen overlapping microRNAs (Figures $6 a$ and b). Notably, the fourteen overlapping genes contain lots of let-7 family members, which intrigued us to explore the effect of linc-ROR on let-7 expression. Besides, we noticed that there is only one different nucleotide sequence between miR-320a and hsa-miR-320b, and microarray showed $>1.5$ fold change of miR-320a, we also further tried to evaluate the effect of linc-ROR on miR-320a expression. 
a

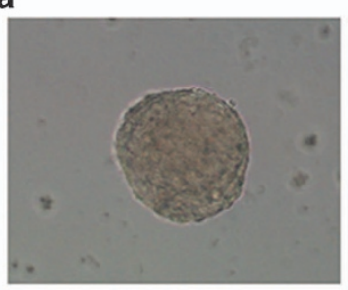

b

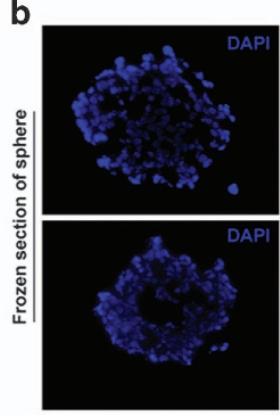

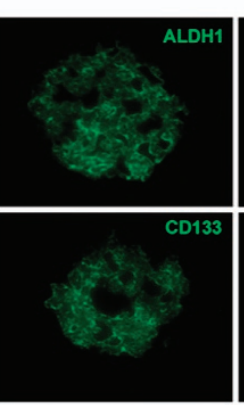

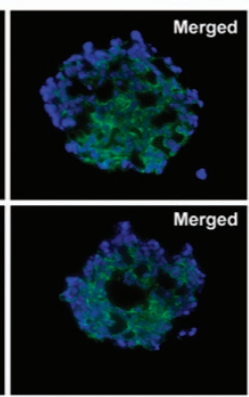

d
C

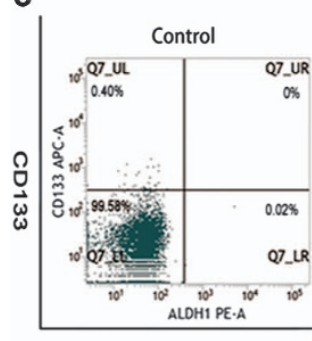

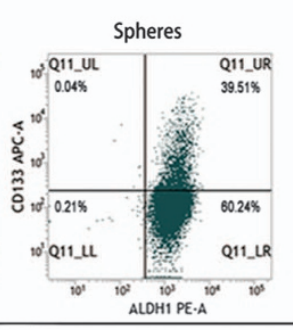

ALDH1
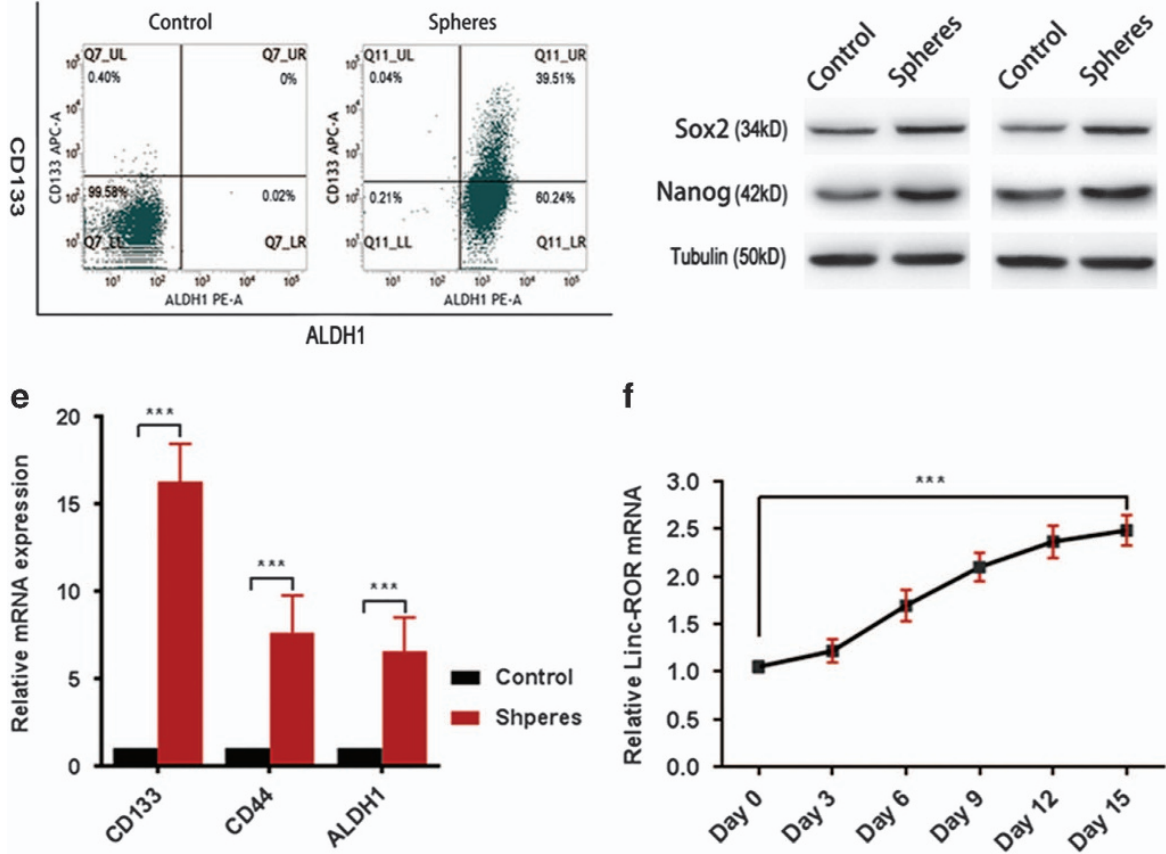

f

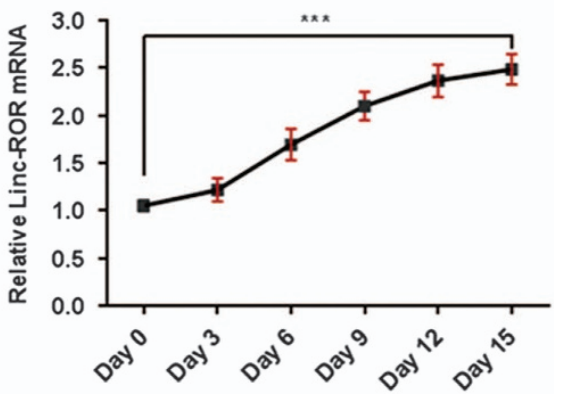

Figure 4. Linc-ROR is over-expressed in pancreatic CSLCs. (a) Representative light microscope images of spheres in PANC-1 cells. (b) Immunofluorescence staining and confocal imaging for CD133 and ALDH1 in PANC-1 spheres. (c) Flow cytometry analysis for CD133 and ALDH1 PANC-1 adherent cells (control) and spheres. (d) Western blot analysis of SOX2 and Nanog in PANC-1 spheres compared with adherent cells as control. (e) Expression of CD133, CD44 and ALDH1 in PANC-1 spheres relative to adherent cells (controls) were determined using qRTPCR. Results are shown as $2 \Delta \Delta C T$. (f) qRT-PCR showing the change of linc-ROR expression levels along with the formation of shperes. Results are shown as ${ }^{2 \Delta \Delta}$ CT. ErROR bars represent the mean \pm S.D. of triplicate experiments. Statistical significance was calculated using the Student's $t$ test or ANOVA tests. ${ }^{* * * P}<0.001$.

Altogether, a total of twenty-seven miRs (Supplementary Table 3) were included for the next step. We first verified the microarray results through re-analyzing the expression of these 27 genes in PANC-1 cells and spheres by using qRT-PCR. Result of PCR were close to that of microarray (Supplementary Figure 1), and the linear regression analysis revealed a correlation between $\mathrm{PCR}$ results and microarray results (Supplementary Figure $2, P<0.001$ ), which indicating that the microarray results were reliable.

Next, we suppressed linc-ROR expression in CSLCs through transfection of siRNA. Compared with controls (siC, transfection of non-targeting siRNA), expression levels of almost all these 27 miRs were increased, and there were 15 miRs increased more than two fold (Figure $6 \mathrm{c}$ ). We employed RNAhybrid to search putative complementary sequences for the seed region of these $15 \mathrm{miRs}$ in linc-ROR, and found there are many potential interaction sites between these miRs and linc-ROR (Supplementary Table 4). To validate the relationship between expression of these miRs and the ceRNA activity of linc-ROR, we further use the RNA immunoprecipitation (RIP) analysis with MS2-binding protein (MS2bp), which specifically binds RNAs that contain MS2binding sequences (MS2bs), when they are co-expressed. Vectors expressing lin-ROR containing MS2bs elements (ROR-MS2bs) connected behind the sequence were constructed, and we cotransfected ROR-MS2bs, Flag-MS2bp, a pool of miRs including the above 15 miRs into PANC-1 cells. Controls were cells transfected with a same amount of the pool of miRs, MS2bs vector, and FlagMS2bp. Subsequently, we performed RIP assay using the ANTIFLAG M2 Affinity Gel. Real-time PCR assays showed that many let7 family members, including hsa-let-7i-5p, hsa-let-7b-5p, hsa-let7e-5p, hsa-let-7e-3p, hsa-let-7b-3p, hsa-let-7c-3p, were enriched in ROR-12*MS2bs RNA in contrast to the controls (Figure 6d). miR-93$5 p$, miR-145-3p, miR-320a, and miR-320b were also found enriched (Figure $6 \mathrm{~d}$ ). Because studies have shown that linc-ROR suppress miR expression through Ago2-related RNA-induced silencing complexes (RISCs), we then confirmed that linc-ROR was recruited to $\mathrm{AGO} 2$ (Figures $6 \mathrm{e}$ and $\mathrm{f}$ ), which was in consistent with previous reports.

\section{DISCUSSION}

Thousands of lincRNAs are discovered in mammalian and play various roles in transcriptional regulation, epigenetics and 
a

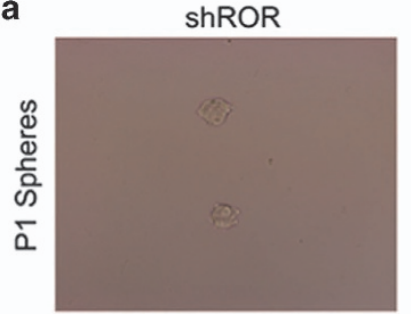

b

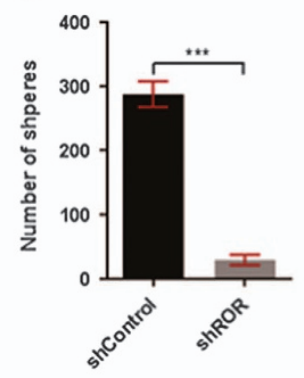

f
C

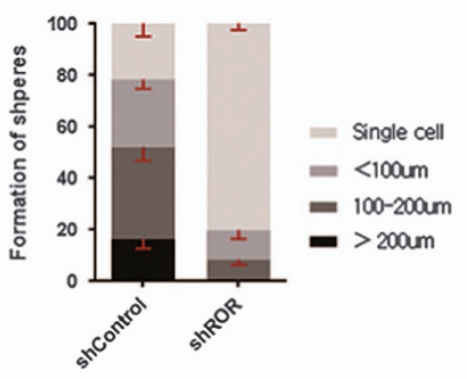

d

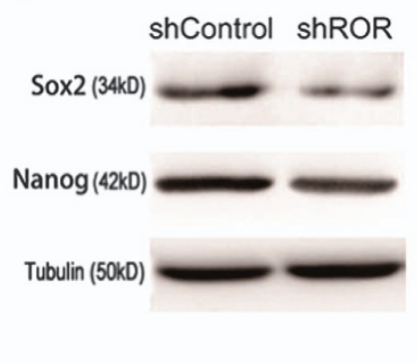

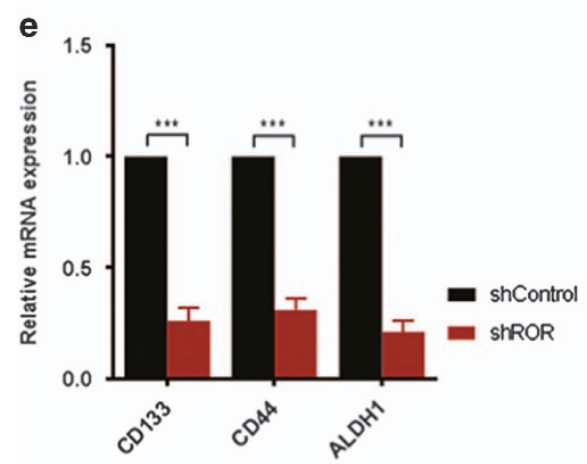
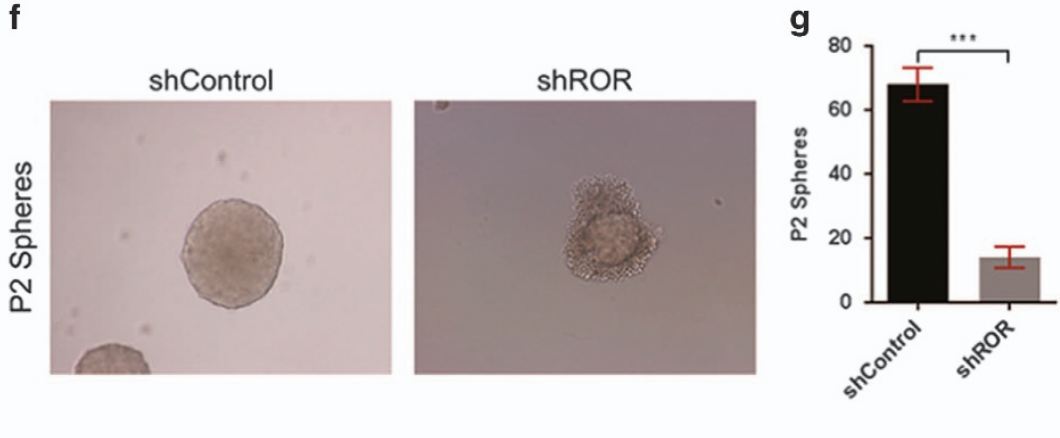

h

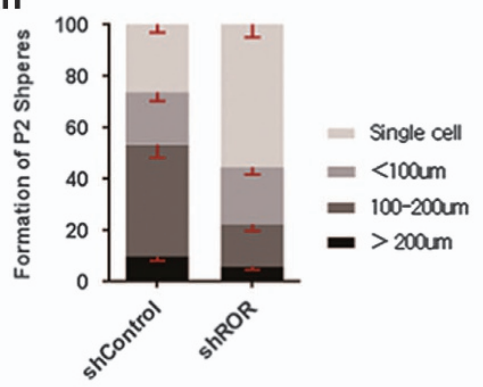

i
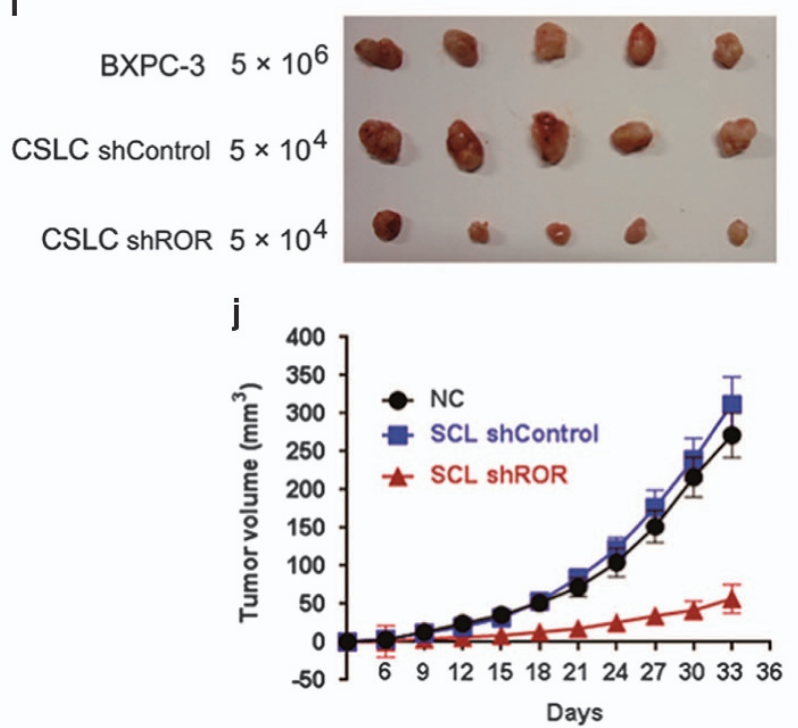

Figure 5. Linc-ROR regulates the CSC properties of stem cell-like pancreatic cancer cells. (a) Representative light microscope images showing spheres derived from PANC-1 cells stably transfected with shRNA-mediated silencing of linc-ROR (shROR). (b) Number of spheres derived from shROR PANC-1 cells compared with shControl PANC-1 cells. (c) Distribution proportion of spheres based on size in shROR PANC-1 cells compared with shControl cells. (d) Western blot analysis of SOX2 and Nanog in shControl PANC-1 sphere cells compared with shROR PANC-1 sphere cells. (e) Expression of CD133, CD44 and ALDH1 in shControl PANC-1 sphere cells relative to shROR PANC-1 sphere cells. (f) Representative light microscope images of P2 spheres derived from shControl and shROR cells of P1 spheres. (g) Comparison of number of P2 spheres derived from P1 spheres. (h) Comparison of size distribution of P2 spheres derived from P1 spheres. (i) The effect of linc-ROR on sphere tumorigenicity was investigated in vivo through injecting the indicated number of PANC-1 cells stable transfected with scramble RNA, CSLC shControl (cells of P1 shperes derived from PANC-1 cells stably transfected with vectors containing scramble RNA), and CSLC shROR (cells of P1 shperes stably derived from PANC-1 cells stably transfected with vectors expression shROR) cells. (j) Tumor volume was determined as described in Materials and methods section. ErROR bars represent the mean \pm S.D. of triplicate experiments. Statistical significance was calculated using the Student's $t$ test or ANOVA tests. ${ }^{* * *} P<0.001$.

occurrence and development of cancer. So far, lots of lincRNAs are researched through chromatin characteristics analysis and mass sequencing, ${ }^{18}$ functional exploration has just begun. Functional studies have showed that several lincRNAs take part in pathogenesis of human cancers, implement as oncogenes or antioncogenes. In this study, we revealed that linc-ROR is commonly overexpressed in PDAC and its overexpression and poor prognosis have a significantly positive correlation, revealing oncogenic function of linc-ROR. The further functional experimenters found that linc-ROR knockdown decreases pancreatic cancer cell proliferation, ability of colony formation, activity and invasion in vitro, and impaired the stem cell-like features of pancreatic cancer cell and tumorigenicity potential in vitro and in vivo. 
a

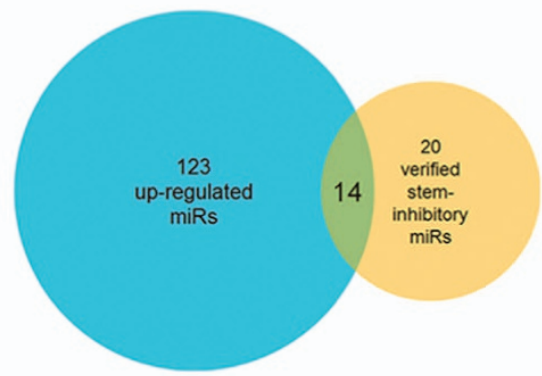

C

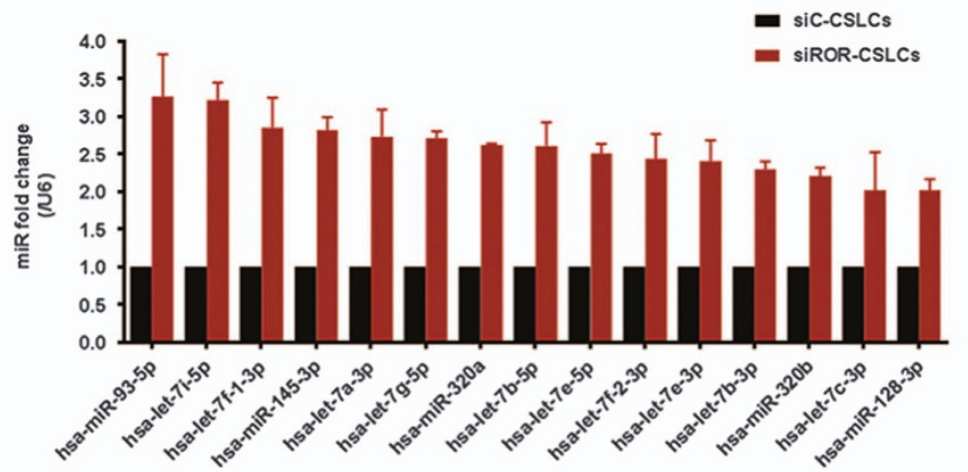

b

\begin{tabular}{ll} 
microRNAs & fold change \\
hsa-miR-7-5p & 5.614895 \\
hsa-miR-93-5p & 3.005904 \\
hsa-let-7c-3p & 2.905138 \\
hsa-miR-128-3p & 2.843654 \\
hsa-let-7f-1-3p & 2.745098 \\
hsa-miR-98-3p & 2.585962 \\
hsa-miR-93-3p & 2.479871 \\
hsa-miR-451a & 2.425678 \\
hsa-miR-320b & 2.362564 \\
hsa-let-7a-3p & 2.254428 \\
hsa-miR-7-1-3p & 2.254428 \\
hsa-let-7i-5p & 2.229239 \\
hsa-let-7f-2-3p & 2.076171 \\
hsa-miR-145-3p & 2.075099 \\
fold change & \\
\hline & $S L C / N C$ \\
\hline 2.0 & \\
\hline
\end{tabular}

d

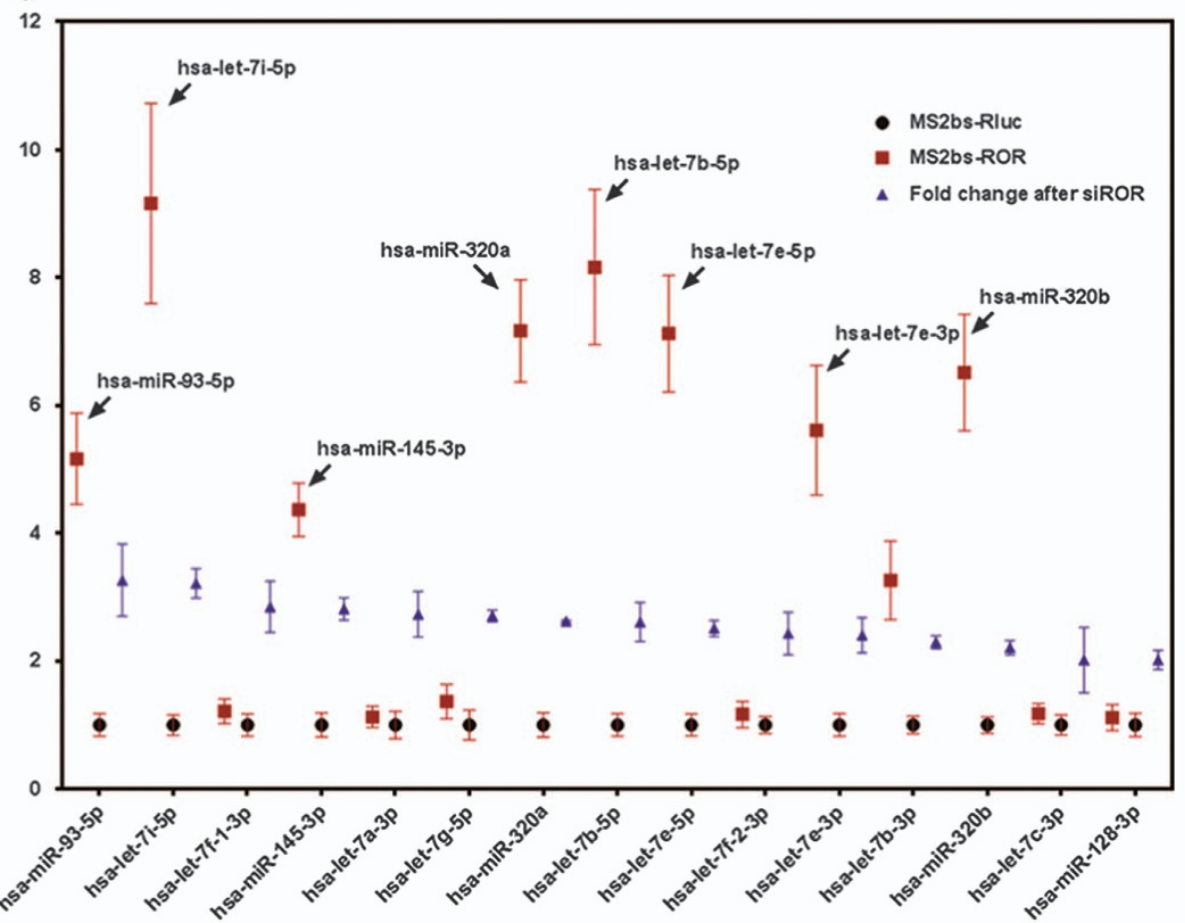

e
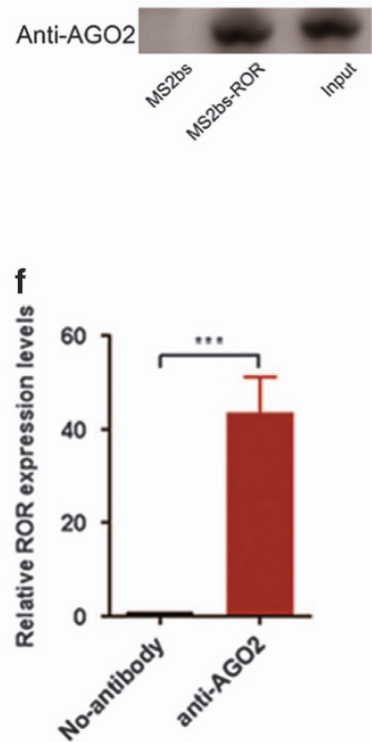

Figure 6. Linc-ROR Functions as an Endogenous microRNA Sponge in pancreatic cancer cells. (a) Total RNAs of PANC-1 cells (NC) and PANC-1 derived stem cell-like cells (SCLCs) were used for a microarray analysis, 123 microRNAs were found decreased more than two-fold in SCLCs compared with PANC-1 cells. By comparing with the confirmed tumor and stemness inhibitory microRNAs, 14 overlaping genes were determined. (b) The heatmap of the 14 determined miRs. The numbers indicated the fold change of gene expression (Fold SCLC/NC) determined by microarray. (c) The effect of linc-ROR on microRNA expression in PANC-1 cells and SCLCs were evaluated by using qRT-PCR. A total of 15 microRNAs elevated more than two-fold after linc-ROR knock-down were shown. (d) The binding ability of the above 15 miRs to linc-ROR full-length transcripts were evaluated by cDNA of linc-ROR combined with MS2-binding sequences (MS2bs) and its binding protein MS2BP-YFP in SCLCs. (e) The binding efficiency of linc-ROR to AGO2 protein in SCLCs were determined by RIP assay, (f) Amount of linc-ROR binding with AGO2 were evaluated by using qRT-PCR. ErROR bars represent the mean \pm S.D. of triplicate experiments. Statistical significance was calculated using the Student's $t$ test or ANOVA tests. ${ }^{* *} P<0.001$. 
LincRNAs have been found to interplay with various proteins, thereby permitting scaffolding functions and combinatorial control. ${ }^{19}$ For example, Xist and Kcnq1ot1 are recognized as interacting with and recruiting histone modification complexes. ${ }^{20}$ LncRNA HOTTIP directly unites WDR5 protein and targets WDR5/ MLL complexes across HOXA, promoting HOXA genes transcription. ${ }^{21}$ During DNA damage, Linc-p21 is up-regulated by p53 and binds hnRNPK to control gene expression. ${ }^{22}$ Another wellknown lincRNA, HOTAIR, can stimulate cancer metastasis through interacting with Polycomb repressive complex 2 to regulate histone H3 lysine 27 methylation. ${ }^{23}$ However, unlike the above mechanism that LncRNA act as protein recruiter and locator, recent data indicate that ncRNAs can regulate gene expression by competing for miRNA binding termed competing endogenous RNA (ceRNA). ${ }^{24}$ Till now, two lincRNAs, linc-MD1 and linc-ROR have been identified to act as ceRNA and, in doing so, inhibits their target microRNAs-mediated messenger RNA (mRNA) degradation., ${ }^{4,12}$

Linc-ROR was first identified as an endogenous sponge inhibiting the differentiation of ESC by targeting miR-145. ${ }^{12}$ Recent studies revealed a tumor-promoting role of linc-ROR in breast cancer, ${ }^{15}$ endometrial cancer. ${ }^{25}$ Most recently, Takahashi et $a .^{14}$ revealed that linc-ROR regulates hypoxia signaling pathways by inhibiting the function of miR-145 in hepatic cancer cells. Importantly, linc-ROR also revealed potential ceRNA activity targeting other miRs exhibits tumor-suppressor activity, such as miR-181a, ${ }^{13,26,27}$ miR-99b, ${ }^{13,28}$ and let-7a-5p. ${ }^{15,29}$ The above findings showed that linc-ROR was a potential carcinogene. In this study, we uncovered a critical role for linc-ROR in pancreatic cancer cell proliferation, showing that linc-ROR knockdown arrests cells at the G1 phase. Besides, our data demonstrated that suppression of lincROR dramatically impaired the expression of mesenchyme markers under the treatment of TGF- $\beta$ and SDF-1. Notably, at the microenvironment level, both TGF- $\beta$ and SDF-1 are major key mediators of the dialogue between cancer and stromal infiltrating cells, ${ }^{30,31}$ which are widespread involved in the regulation of cancer cell proliferation, differentiation, invasion, and inflammation. ${ }^{32}$ Therefore, it seems that linc-ROR would continue to play a role in tumor progression under complex tumor microenvironment.

Cancer stem cells (CSCs) are stirring field for cancer studies, and provide a novel target for tumor treatment. Cancer stem cells are defined as rare cells in cancer tissues with indefinite potential for self-renewal that drives tumorigenesis. ${ }^{33,34}$ As the identification of CSCs or cancer stem-like cells (CSLCs) in pancreatic cancer, ${ }^{35,36}$ pancreatic CSCs/CSLCs have emerged as a possible, attractive explanation for the highly incorrigible therapy resistance of pancreatic cancer. ${ }^{37}$ Thus the molecular mechanism contributing to the maintenance of pancreatic CSCs/CSLSc properties have been an emerging focus of recent research. As linc-ROR was shown to play an important part in preserving the pluripotency of human embryo stem cells, ${ }^{13}$ here we studied emphatically on the role of linc-ROR in maintaining the pancreatic CSLCs properities. Compliance with its role in iPS cells, we found that linc-ROR expression relates to stemness in pancreatic cancer cell. Our data showed that knockdown of linc-ROR reduced sphere formation, diminished CSC marker expression, and impaired tumorigenesis.

MicroRNAs have been implicated in the regulation of CSC properties including cell-cycle, differentiation, migration, invasion and EMT, which contribute to improve Initiation and metastasis of tumor. ${ }^{38,39}$ Except miR-145 which was identified as a target of lincROR, Hou et al. ${ }^{15}$ discovered that linc-ROR increases breast cancer cells EMT via sponge mir-205; meanwhile, their data also demonstrated that let-7a-5p mimics treatment decreased about 20 percent of Rluc activity in cells transfected with luciferase reporter gene containing linc-ROR complementary DNA (cDNA) compared with controls. ${ }^{15}$ Wang et al. ${ }^{21}$ also reported that miR-181a-5p and miR-99b-3p expression were enhanced in lincROR transcript besides miR-145-5p. The above findings suggested that linc-ROR could sponge some different microRNAs. To explore how linc-ROR function during formation of pancreatic CSLCs, we picked miRs with potential importance in inhibiting CSC properties in pancreatic CSLCs through comparing the microarray data with CSC-inhibiting miRs reported in published articles, and we further selectively up-regulated miRs following linc-ROR knockdown from these picked miRs. At last, we identified that several CSCinhibiting miRs, including several members of let-7 family, miR-93$5 p$, miR-320a, miR-320b, and miR-145-3p could be enriched in lincROR transcript. It should be noted that the degree of enchainment of miRs were not consistent with the levels of up-regulation of these miRs mediated by linc-ROR known-down. One possible assumption for this inconsistence might be that linc-ROR could regulate microRNA expression through indirect pathway. For example, miR-320 demonstrated a inhibitory effect on Wnt/beta-catenin signaling pathway, ${ }^{40}$ which regulates the expression of several CSCrelated microRNA such as miR-34, ${ }^{41}$ miR-302, ${ }^{42}$ and let- $7 .{ }^{43}$ The down-regulation of other miRs might be caused by other cellular process mediated by linc-ROR via sponge several key microRNAs.

To summarize, the present work identifies linc-ROR as a novel potential oncogene in pancreatic cancer through contributing to PDAC proliferation, dedifferentiation, and stemness. We also identified some potential target microRNAs of linc-ROR in maintaining the CSC properties of pancreatic CSLCs. Owing to the vital role of these features in PDAC, we propose linc-ROR as a promising target of new therapies for this this intractable malignancy.

\section{MATERIALS AND METHODS}

\section{Cell culture}

The human pancreatic cancer cell lines PANC-1 and SW1990 were gained from American Type Culture Collection (Manassas, VA) and grown in DMEM (Gibco, NY, USA) supplemented with $10 \% \mathrm{FBS}, 100 \mathrm{U} / \mathrm{ml}$ penicillin $(100 \mathrm{U} / \mathrm{ml}$, Sigma, St Louis, MO, USA) and $100 \mathrm{U} / \mathrm{ml}$ streptomycin (100 $\mu \mathrm{g} / \mathrm{ml}$, Sigma), in a humidified $5 \% \mathrm{CO} 2$ atmosphere at $37^{\circ} \mathrm{C}$. To enrich the CSC-like cells, chemotherapy sorting and suspension culture supporting proliferation of undifferentiated cells were adopted. ${ }^{36,44-46}$ Briefly, $1 \times 10^{6}$ PANC- 1 cells were given subcutaneous injection into the low right axilla nude mice. When the tumor volume reached $\sim 250 \mathrm{~mm}^{3}$, the tumors were treated with $100 \mathrm{mg} / \mathrm{kg}$ gemcitabine twice weekly. Xenograft were removed after treated with gemcitabine for 4 weeks, excised, and evenly re-implanted in additional nude mice treated with $100 \mathrm{mg} / \mathrm{kg}$ gemcitabine twice weekly as well. After 4 weeks, the second passage xenograft were removed, excised, washed in Hanks three times, and shredded in serum-free DMEM added with $200 \mathrm{U} / \mathrm{ml}$ type IV collagenase (Sigma). Then the cell suspension was thrice filtered through $70 \mu \mathrm{M}$ nylon filters, followed by $800 \mathrm{r} / \mathrm{min}$ centrifugation. Cells were re-suspended in DMEM medium containing penicillin, streptomycin, $0.4 \%$ bovine serum albumin (Sigma), B-27 supplement (1:50; Gibco, Carlsbad, CA, USA), $20 \mathrm{ng} / \mathrm{ml}$ epidermal growth factor (Invitrogen, Carlsbad, CA) and $20 \mathrm{ng} /$ $\mathrm{ml}$ basic fibroblast growth factor (Invitrogen) in low-adhesion culture bottle (Corning, NY, USA) at a density of $10^{4}$ cells $/ \mathrm{ml}$.

\section{Vector construction and virus infection}

The pMKO.1-puro retroviral vector was purchased from Sigma. The scrambled control shRNA and linc-ROR shRNA were implanted into the pMKO.1-puro vector: linc-ROR, forward, 5'-CCGGAGGAGAGGAAGCCT GAGAGTCTCGAGACTCTCAGGCTTCCTCTCCTIIITTG-3' and reverse, 5'-AATTCAAAAAAGGAGAGGAAGCCTGAGAGTCTCGAGACTCTCAGGCTTCCTCTCCT-3'; scrambled control shRNA, forward 5'-CCGGTITCTCCGAACGTGTCACGTC TCGAGACGTGACACGTTCGGAGAATITTG-3'and reverse, 5'-AATTCAAAAAG TTCTCCGAACGTGTCACGTCTCGAGACGTGACACGTTCGGAGAA-3'. The complementary DNA of linc-ROR was purchased from RiboBio Biological Company (Guangzhou, China), and was implanted into the EcoR1 site of pcDNA3 (+) vector. packaging virus, infecting cells and selecting puromycin-resistant cells were described in previous research. ${ }^{47}$

\section{RNA isolation and quantitative real-time PCR}

Total RNA of pancreatic cancer tissue or cells was extracted, then was converted to CDNA were performed, as previously described. ${ }^{48}$ Quantitative real time PCR for quantification of linc-ROR, $\beta$-actin (as internal control) and other mRNA was executed on a Roche Light-Cycler system (Roche, 
Basel, Switzerland) by using SYBR Green reaction mix (Qiagen, Foster City, CA, USA) (PCR primers sequences are supplied in Supplementary Materials). Quantitative miRNA levels were determined using real-time RT-PCR with the Applied Biosystems 7900 HT Sequence Detection System (Thermo Fisher Scientific, Waltham, MA, USA), TaqManH Gene Expression Assay (Thermo Fisher Scientific) for human let-7c-3p (assay ID 002479), hsalet-7f-1-3p (assay ID 002417), hsa-let-7a-3p (assay ID 002307), hsa-let-7i-5p (assay ID 002221), hsa-let-7f-2-3p (assay ID 002418), hsa-let-7b-3p (assay ID 002404), hsa-let-7g-5p (assay ID 002282), hsa-let-7e-3p (assay ID 002407), hsa-let-7i-3p (assay ID 002172), hsa-let-7b-5p (assay ID 002619), hsa-let-7g$3 p$ (assay ID 002118), hsa-let-7c-5p (assay ID 000379), hsa-let-7e-5p (assay ID 002406), hsa-let-7d-5p (assay ID 002283), hsa-let-7f-5p (assay ID 000382), hsa-let-7d-3p (assay ID 001178), hsa-let-7a-5p (assay ID 000377), hsamiR-320a (assay ID 002277), hsa-miR-320b (assay ID 002844), has-miR-7-5p (assay ID 000268), has-miR-7-1-3p (assay ID 001338), has-miR-93-5p (assay ID 001090), has-miR-93-3p (assay ID 002139), has-miR-128-3p (assay ID 002216), has-miR-451a (assay ID 001105), and U6 snRNA (assay ID 001973) as an endogenous control. Reverse transcriptase reactions were performed by $10 \mathrm{ng}$ of total RNA wich TaqMan Universal PCR Master Mix No AmpErase (Thermo Fisher Scientific) and respective TaqManH reagents. Real-time PCR was done in a total volume of $20 \mu \mathrm{l}$ reaction mixture according to the manufacture's protocol. The reactions were incubated in 96-well optical plates at $95^{\circ} \mathrm{C}$ for $10 \mathrm{~min}$, and then followed by 40 cycles of $95^{\circ} \mathrm{C}$ for $15 \mathrm{~s}$ and $60^{\circ} \mathrm{C}$ for $1 \mathrm{~min}$. Relative gene expression levels of mRNA and miRNAs were determined using the ${ }^{\Delta \Delta} \mathrm{Ct}$-method.

\section{Immunoblotting}

Immunoblotting were executed as described previously. ${ }^{47}$ Simply, cells were washed with PBS and lysed in RIPA buffer (Invitrogen) added protease inhibitor (Sigma). Protein concentration of each sample was calculate by BCA protein assay kit (Thermo Fisher Scientific) to equal protein loading. Equivalent protein were underwent to SDS-PAGE, shifted to polyvinylidene fluoride membrane, interdicted in 5\% skim milk for about $2 \mathrm{~h}$ at about $25^{\circ} \mathrm{C}$, then checked with relative primary antibodies. The following antibodies were used for analysis: anti-E-cadherin, anti$\mathrm{N}$-cadherin, anti-Vimentin and anti-SOX2 (Abcam plc, Cambridge, UK), anti-Nanog (Cell Signaling, Beverly, MA, USA), anti- $\beta$-actin (Sigma) and anti$\beta$-tubulin (BD Biosciences, CA, USA). $\beta$-actin and $\beta$-tubulin was served as loading controls. Horseradish Peroxidase (HRP) Secondary Antibodies (Abcam) and an ECL Chemiluminescence Detection Kit (Thermo Fisher Scientific) were used to test combined antibody.

\section{Cell proliferation analysis}

Cell Proliferation Kit I (MTT) (Sigma) was used to test cell proliferation consistent with the manufacturer's instructions. Concisely, cells were adhered and grown in 96-well dishes with a density of $5 \times 10^{3}$ cells/well overnight. The proliferation curves of cells, involving cultured cells in three days, were formulated by measuring absorbance at $570 \mathrm{~nm}$.

\section{Flow cytometry analysis}

Single cell suspensions with PBS were washed twice and $2 \times 10^{6}$ cells/tube were incubated cell cycle detection reagent or fluorescent-labeled monoclonal antibodies at $4{ }^{\circ} \mathrm{C}$ in dark for $30 \mathrm{~min}$. For cell cycle analysis, propidium iodide ( $\mathrm{Pl}$, Molecular Probes, BD Biosciences) was used in staining. For detecting CSC markers, the ALDH1-PE and CD133-APC (BD Biosciences) antibodies were used. Cells were washed twice with $2 \mathrm{ml}$ PBS, and then re-suspended in $200 \mathrm{ml} \mathrm{PBS}$, last analyzed using Flow Cytometer (FACSVerse, BD Biosciences).

\section{Migration and invasion assays}

For cell migration assays, pancreatic cancer cells were seeded into 6-well culture plates by $2 \times 10^{5}$ cells/well. After $48 \mathrm{~h}$, the attached cells were lightly scarred using a sterile $10-\mu \mathrm{l}$ tip and was cultured for an additional $12 \mathrm{~h}$ by serum-free medium. Invasion experiments were implemented by using $8-\mu \mathrm{m}$ pore size Transwell plates (Corning). Briefly, $5 \times 10^{4}$ cells resuspended in serum-free medium was added to the upper wells. For promoting migration or invasion, complete medium was added to the under wells. After cultivation for $24-48 \mathrm{~h}$, cells, non-penetrating through the membrane, were wiped by a swab, and the cells that were adhered to the bottom surfaces of the membrane were dyed using $0.1 \%$ crystal violet for $20 \mathrm{~min}$. The number of cells adhered to the bottom surface was counted under the microscope.
Transfection of siRNA

siRNA targeting linc-ROR were selected and transfected as described by Takahashi et al. ${ }^{14}$ siRNA against linc-ROR: 5'-GGAGAGGAAGCCTGAGAGT-3'; non-targeting control (siC) siRNA were purchased from Dharmacon (Lafayette, CO, USA). miRNA were obtained from RiboBio (Guangzhou, China). Transfections were completed with Lipofectamine 2000 (Invitrogen) for $48 \mathrm{~h}$ before further experiments.

\section{RNA-binding protein immunoprecipitation (RIP) assay}

The MS2bp-MS2bs-based RIP assay was achieved consistent with previous published paper ${ }^{13}$ by the EZ-Magna RIP ${ }^{\text {TM }}$ Kit (Merck Millipore, Munich, Germany). Briefly, cells of spheres $\left(5 \times 10^{6}\right)$ were seeded into $100 \mathrm{~mm}$ plate and incubated overnight then co-transfected with $20 \mu \mathrm{g}$ linc-ROR-MS2bs overexpressing vectors (pcDNA3-ROR-MS2bs) or blank control vectors with Renilla luciferase inserts (pcDNA3-MS2bs-Rluc), $5 \mu \mathrm{g}$ FLAG-MS2bp overexpressing plasmid and indicated microRNA minics molecules (100 nM for each minics) with Lipofectamine 2000. After $48 \mathrm{~h}, 1 \times 10^{7}$ cells were collected and lysed with RIP lysis buffer supplemented with $80 \mathrm{U} / \mathrm{ml}$ ribonuclease Inhibitor (RNasin, Promega, Madison, WI, USA). ANTI-FLAG M2 Magnetic Beads were used for isolation FLAG-MS2bp-MS2bs-linc-ROR complex. To evaluating the enrichment of miRNAs in linc-ROR, the ANTIFLAG M2 Affinity Gel (Sigma) was used in RIP assay. The complexes of RNA and RNA-binding proteins were then treated with Trizol (Invitrogen), RNA was extracted and further detected by PCR.

\section{Sphere-formation assays}

Single cell suspensions $\left(5 \times 10^{3}\right.$ cells $\left./ \mathrm{ml}\right)$ were seeded into low adhesion six-well plates (Corning) and incubated in modified DMEM as described in 'Cell culture' section without supplementation of serum. Media was replaced every 3 days. Spheres were counted after 14 days (passage one, P1). The number of secondary spheres (passage two, P2) formed following 10-days incubation after scattering was counted.

\section{Patients and clinical specimens}

Fresh frozen tumor and para-tumor tissues were gained during resection of pancreatic cancer in Sun Yat-sen Memorial Hospital, The tissues were quickfrozen in liquid-nitrogen and deposited at $-80^{\circ} \mathrm{C}$ until tested. Formalin-fixed and paraffin-embedded tissues were obtained from the pathology department. The patients should sign informed consent before sample collection. Furthermore the committees of hospital should approve ethical review of the research. The patients had not received chemo- or radio-therapy before operation. The histological of each primary sample was estimated through pathological review, only invasive ductal adenocarcinomas were included, and frozen samples with $<70 \%$ tumors cellularity were excluded.

\section{In vivo tumorigenesis assay}

Five weeks-old male BALB/c nude mice were achieved from the Laboratory Animal Center, Zhongshan Medical School of Sun Yat-sen University and raised in laminar flow cabinets under specific pathogen-free (SPF) conditions. All procedures of experiment linking to animals were consistent with the Guide for the Care and Use of Laboratory Animals and were implemented along with the institutional ethical guidelines for animal experiment. The study protocol was also approved by the Committee on the Use of Live Animals in Teaching and Research, Sun Yat-sen Memorial Hospital, Sun Yat-sen University.

PANC-1 cells and PANC-1-derived sphere (shperes of 100-200 $\mu \mathrm{m}$ in diameter were selected) were collected, dissociated to single cell by trypsinEDTA, washed in PBS, counted and injected into the low right axilla of each mice. Tumor size was measured with the following formula: volume $=\left(L \times W^{2}\right) / 2$, where $L$ and $W$ are the longest and shortest diameters, respectively. Mice were killed when the average $L$ of any group reached about $1 \mathrm{~cm}$.

\section{ACKNOWLEDGEMENTS}

Supported by the National Natural Science Foundation of China, Nos. 81370059, 81000917 and 81402213; Guangdong Science and Technology Department, Nos. S2012010008934, 2014A030313044, 2014A030311047, 2015A030310489, 2016A030313340 and 2016A030313296; and Foundation of '5010' project of Sun Yat-sen University, No. 2012007. 


\section{COMPETING INTERESTS}

The authors declare no conflict of interest.

\section{REFERENCES}

1 Mercer TR, Dinger ME, Mattick JS. Long non-coding RNAs: insights into functions. Nat Rev Genet 2009; 10: 155-159.

2 Khaitan D, Dinger ME, Mazar J, Crawford J, Smith MA, Mattick JS et al. The melanoma-upregulated long noncoding RNA SPRY4-IT1 modulates apoptosis and invasion. Cancer Res 2011; 71: 3852-3862.

3 Loewer S, Cabili MN, Guttman M, Loh YH, Thomas K, Park IH et al. Large intergenic non-coding RNA-RoR modulates reprogramming of human induced pluripotent stem cells. Nat Genet 2010; 42: 1113-1117.

4 Cesana M, Cacchiarelli D, Legnini I, Santini T, Sthandier O, Chinappi M et al. A long noncoding RNA controls muscle differentiation by functioning as a competing endogenous RNA. Cell 2011; 147: 358-369.

5 Ginger MR, Shore AN, Contreras A, Rijnkels M, Miller J, Gonzalez-Rimbau MF et al. A noncoding RNA is a potential marker of cell fate during mammary gland development. Proc Natl Acad Sci USA 2006; 103: 5781-5786.

6 Zhang H, Chen Z, Wang X, Huang Z, He Z, Chen Y. Long non-coding RNA: a new player in cancer. J Hematol Oncol 2013; 6: 37.

7 Seton-Rogers S. Non-coding RNAs: the cancer X factor. Nat Rev Cancer 2013; 13: 224-225.

8 Karreth FA, Pandolfi PP. ceRNA cross-talk in cancer: when ce-bling rivalries go awry. Cancer Discov 2013; 3: 1113-1121.

9 Tay Y, Rinn J, Pandolfi PP. The multilayered complexity of ceRNA crosstalk and competition. Nature 2014; 505: 344-352.

10 Kumar MS, Armenteros-Monterroso E, East P, Chakravorty P, Matthews N, Winslow MM et al. HMGA2 functions as a competing endogenous RNA to promote lung cancer progression. Nature 2014; 505: 212-217.

11 Wang J, Liu X, Wu H, Ni P, Gu Z, Qiao Y et al. CREB up-regulates long non-coding RNA, HULC expression through interaction with microRNA-372 in liver cancer. Nucleic Acids Res 2010; 38: 5366-5383.

12 Cheng EC, Lin H. Repressing the repressor: a lincRNA as a MicroRNA sponge in embryonic stem cell self-renewal. Dev Cell 2013; 25: 1-2.

13 Wang $Y, X u Z$, Jiang J, Xu C, Kang J, Xiao L et al. Endogenous miRNA sponge lincRNA-RoR regulates Oct4, Nanog, and Sox2 in human embryonic stem cell self-renewal. Dev Cell 201z3; 25: 69-80.

14 Takahashi K, Yan IK, Haga H, Patel T. Modulation of hypoxia-signaling pathways by extracellular linc-RoR. J Cell Sci 2014; 127(Pt 7): 1585-1594.

15 Hou P, Zhao Y, Li Z, Yao R, Ma M, Gao Y et al. LincRNA-ROR induces epithelial-tomesenchymal transition and contributes to breast cancer tumorigenesis and metastasis. Cell Death Dis 2014; 5: e1287.

16 Gunaratne $\mathrm{PH}$. Embryonic stem cell microRNAs: defining factors in induced pluripotent (iPS) and cancer (CSC) stem cells? Curr Stem Cell Res Ther 2009; 4: 168-177.

17 Herreros-Villanueva M, Bujanda L, Billadeau DD, Zhang JS. Embryonic stem cell factors and pancreatic cancer. World J Gastroenterol 2014; 20: 2247-2254.

18 Guttman M, Amit I, Garber M, French C, Lin MF, Feldser D et al. Chromatin signature reveals over a thousand highly conserved large non-coding RNAs in mammals. Nature 2009; 458: 223-227.

19 Ulitsky I, Bartel DP. lincRNAs: genomics, evolution, and mechanisms. Cell 2013; 154: 26-46.

20 Hung T, Chang HY. Long noncoding RNA in genome regulation: prospects and mechanisms. RNA Biol 2010; 7: 582-585.

21 Wang KC, Yang YW, Liu B, Sanyal A, Corces-Zimmerman R, Chen Y et al. A long noncoding RNA maintains active chromatin to coordinate homeotic gene expression. Nature 2011; 472: 120-124.

22 Huarte M, Guttman M, Feldser D, Garber M, Koziol MJ, Kenzelmann-Broz D et al. A large intergenic noncoding RNA induced by p53 mediates global gene repression in the p53 response. Cell 2010; 142: 409-419.

23 Gupta RA, Shah N, Wang KC, Kim J, Horlings HM, Wong DJ et al. Long non-coding RNA HOTAIR reprograms chromatin state to promote cancer metastasis. Nature 2010; 464: 1071-1076.

24 Salmena L, Poliseno L, Tay Y, Kats L, Pandolfi PP. A ceRNA hypothesis: the Rosetta Stone of a hidden RNA language? Cell 2011; 146: 353-358.

25 Zhou X, Gao Q, Wang J, Zhang X, Liu K, Duan Z. Linc-RNA-RoR acts as a 'sponge' against mediation of the differentiation of endometrial cancer stem cells by microRNA-145. Gynecol Oncol 2014; 133: 333-339.

26 Pichler M, Winter E, Ress AL, Bauernhofer T, Gerger A, Kiesslich T et al. miR-181a is associated with poor clinical outcome in patients with colorectal cancer treated with EGFR inhibitor. J Clin Pathol 2014; 67: 198-203.
27 Zhai XF, Fang FF, Liu Q, Meng YB, Guo YY, Chen Z. MiR-181a contributes to bufalin-induced apoptosis in PC-3 prostate cancer cells. BMC Complement Altern Med 2013; 13: 325.

28 Wei F, Liu Y, Guo Y, Xiang A, Wang G, Xue X et al. miR-99b-targeted mTOR induction contributes to irradiation resistance in pancreatic cancer. Mol Cancer 2013; 12: 81.

29 Bussing I, Slack FJ, Grosshans H. let-7 microRNAs in development, stem cells and cancer. Trends Mol Med 2008; 14: 400-409.

30 Neuzillet C, de Gramont A, Tijeras-Raballand A, de Mestier L, Cros J, Faivre S et al. Perspectives of TGF-beta inhibition in pancreatic and hepatocellular carcinomas. Oncotarget 2014; 5: 78-94.

31 Koshiba T, Hosotani R, Miyamoto Y, Ida J, Tsuji S, Nakajima S et al. Expression of stromal cell-derived factor 1 and CXCR4 ligand receptor system in pancreatic cancer: a possible role for tumor progression. Clin Cancer Res 2000; 6: 3530-3535.

32 Lunardi S, Muschel RJ, Brunner TB. The stromal compartments in pancreatic cancer: are there any therapeutic targets? Cancer Lett 2014; 343: 147-155.

33 Clarke MF, Dick JE, Dirks PB, Eaves CJ, Jamieson CH, Jones DL et al. Cancer stem cells--perspectives on current status and future directions: AACR workshop on cancer stem cells. Cancer Res 2006; 66: 9339-9344.

34 Gong C, Bauvy C, Tonelli G, Yue W, Delomenie C, Nicolas V et al. Beclin 1 and autophagy are required for the tumorigenicity of breast cancer stem-like/ progenitor cells. Oncogene 2013; 32: 2261-2272.

35 Li C, Heidt DG, Dalerba P, Burant CF, Zhang L, Adsay V et al. Identification of pancreatic cancer stem cells. Cancer Res 2007; 67: 1030-1037.

36 Hermann PC, Huber SL, Herrler T, Aicher A, Ellwart JW, Guba M et al. Distinct populations of cancer stem cells determine tumor growth and metastatic activity in human pancreatic cancer. Cell Stem Cell 2007; 1: 313-323.

37 Okada M, Shibuya K, Sato A, Seino S, Suzuki S, Seino M et al. Targeting the K-RasJNK axis eliminates cancer stem-like cells and prevents pancreatic tumor formation. Oncotarget 2014; 5: 5100-5112.

38 Takahashi RU, Miyazaki $\mathrm{H}$, Ochiya $\mathrm{T}$. The role of microRNAs in the regulation of cancer stem cells. Front Genet 2014; 4: 295.

39 Garg M. MicroRNAs, stem cells and cancer stem cells. World J Stem Cells 2012; 4: 62-70.

40 Hsieh IS, Chang KC, Tsai YT, Ke JY, Lu PJ, Lee KH et al. MicroRNA-320 suppresses the stem cell-like characteristics of prostate cancer cells by downregulating the Wnt/beta-catenin signaling pathway. Carcinogenesis 2013; 34: 530-538.

41 Tamura M, Uyama M, Sugiyama Y, Sato M. Canonical Wnt signaling activates miR-34 expression during osteoblastic differentiation. Mol Med Rep 2013; 8: 1807-1811.

42 Brautigam C, Raggioli A, Winter J. The Wnt/beta-catenin pathway regulates the expression of the miR-302 cluster in mouse ESCS and P19 cells. PLoS One 2013; 8: e75315.

43 Cai WY, Wei TZ, Luo QC, Wu QW, Liu QF, Yang M et al. The Wnt-beta-catenin pathway represses let-7 microRNA expression through transactivation of Lin28 to augment breast cancer stem cell expansion. J Cell Sci 2013; 126(Pt 13): 2877-2889.

44 Jimeno A, Feldmann G, Suarez-Gauthier A, Rasheed Z, Solomon A, Zou GM et al. A direct pancreatic cancer xenograft model as a platform for cancer stem cell therapeutic development. Mol Cancer Ther 2009; 8: 310-314.

45 Li L, Li B, Shao J, Wang X. Chemotherapy sorting can be used to identify cancer stem cell populations. Mol Biol Rep 2012; 39: 9955-9963.

46 Du Z, Qin R, Wei C, Wang M, Shi C, Tian R et al. Pancreatic cancer cells resistant to chemoradiotherapy rich in 'stem-cell-like' tumor cells. Dig Dis Sci 2011; 56: 741-750.

47 Tan L, Ye X, Zhou Y, Yu M, Fu Z, Chen R et al. Macrophage migration inhibitory factor is overexpressed in pancreatic cancer tissues and impairs insulin secretion function of beta-cell. J Transl Med 2014; 12: 92.

48 Ye Y, Chen J, Zhou Y, Fu Z, Zhou Q, Wang Y et al. High expression of AFAP1-AS1 is associated with poor survival and short-term recurrence in pancreatic ductal adenocarcinoma. J Transl Med 2015; 13: 137.

This work is licensed under a Creative Commons Attribution 4.0 International License. The images or other third party material in this article are included in the article's Creative Commons license, unless indicated otherwise in the credit line; if the material is not included under the Creative Commons license, users will need to obtain permission from the license holder to reproduce the material. To view a copy of this license, visit http://creativecommons.org/licenses/ by/4.0/

(c) The Author(s) 2017

Supplementary information accompanies the paper on the Cell Death Discovery website website (http://www.nature.com/cddiscovery) 